\title{
In vitro diagnosis of allergy: how to interpret IgE antibody results in clinical practice
}

\author{
Staffan Ahlstedt ${ }^{a, b, *}$, Clare S. Murray ${ }^{c}$ \\ a National Institute of Environmental Medicine, Centre for Allergy Research, \\ Karolinska Institute, Stockholm, Sweden \\ b Phadia AB, Uppsala, Sweden \\ c University of Manchester, North West Lung Centre, South Manchester University \\ Hospitals NHS Trust, Manchester, UK
}

Received 27 February 2006; accepted 30 May 2006

\begin{tabular}{|c|c|}
\hline $\begin{array}{l}\text { KEYWORDS } \\
\text { Allergy; } \\
\text { IgE antibody tests; } \\
\text { In vitro; } \\
\text { Diagnocis }\end{array}$ & 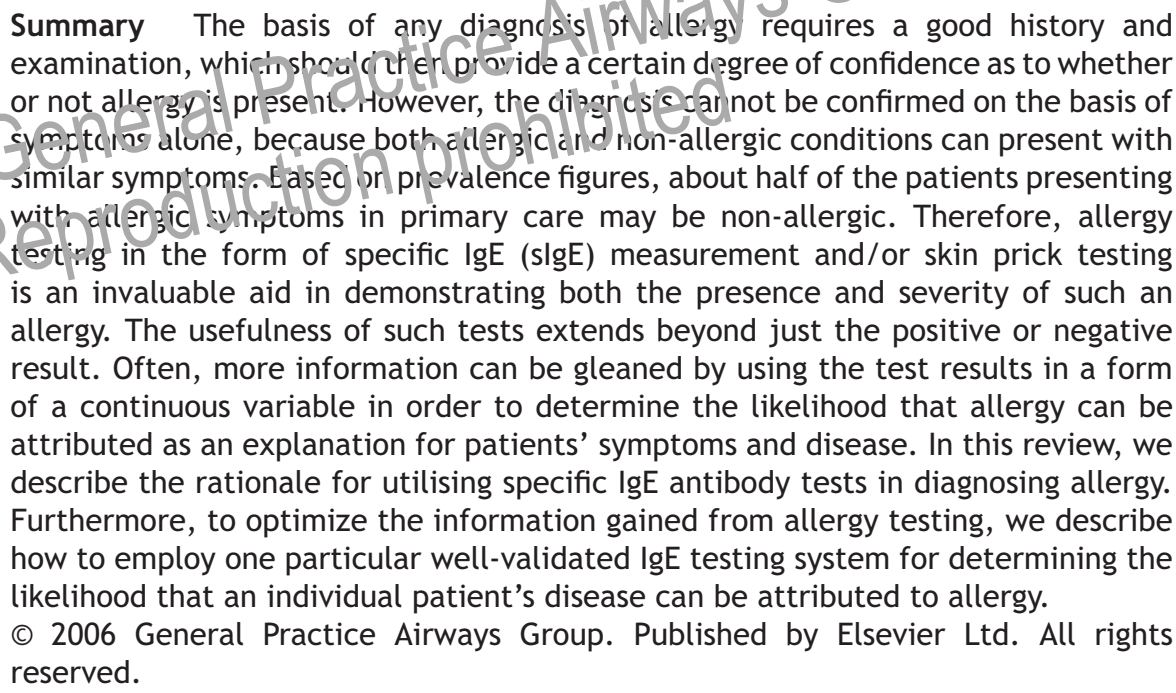 \\
\hline
\end{tabular}

\section{Contents}

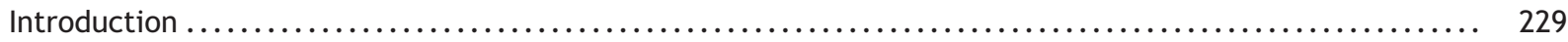

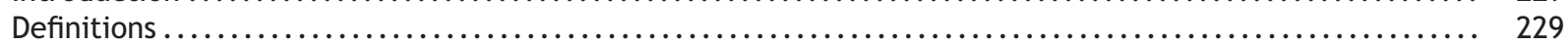

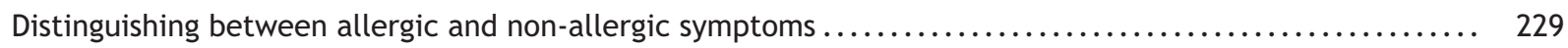

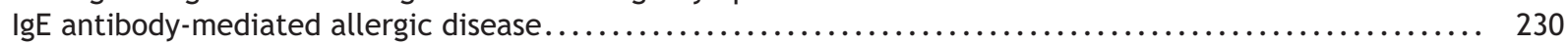

\footnotetext{
* Corresponding author. Tel.: +46 181650 17; fax: +46 18140358 .

E-mail address: staffan.ahlstedt@phadia.com (S. Ahlstedt).
} 


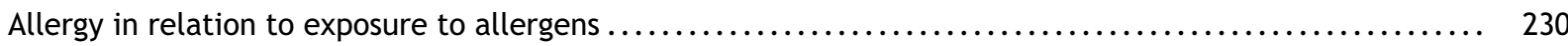

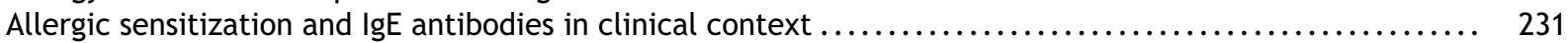

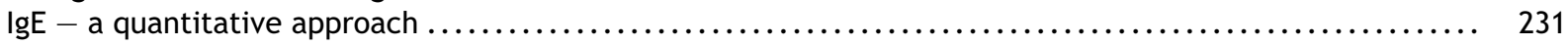

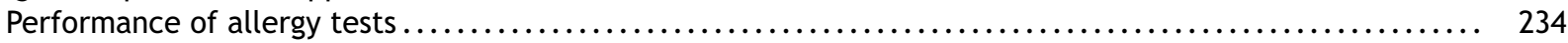

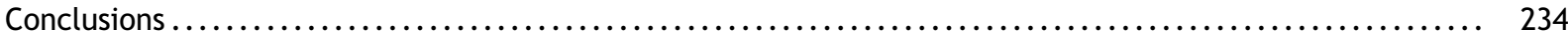

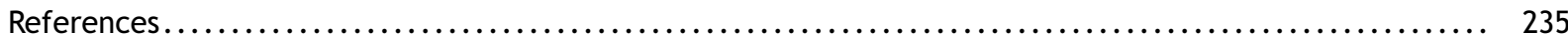

\section{Introduction}

Allergic diseases present as a multitude of symptoms and signs, which are often very difficult to distinguish from similar clinical conditions which are non-allergic in origin. Within primary care, as many as $60-70 \%$ of conditions commonly regarded as allergic may be of non-allergic aetiology [1]. For example, seasonal or perennial respiratory symptoms that resemble allergy may actually be due to infections, vasomotor conditions, anatomical conditions, or chronic obstructive pulmonary disease (COPD). To date there are no prospective studies that have specifically aimed to differentiate between allergic and non-allergic rhinitis on the basis of clinical symptoms, signs, and physical examination. Furthermore, any minimum level of testing necessary to confirm or exclude a diagnosis of allergic rhinitis has not been established [2].

\section{Definitions}

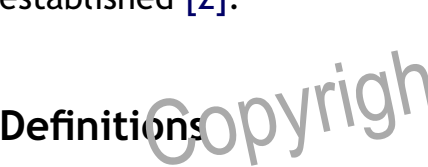

It is essential to define the terms used when discussing allergic disease. The definitions of several key terms are given in Box 1.

\section{Distinguishing between allergic and non-allergic symptoms}

Patients are often started on empirical therapy based on the history alone, without employing diagnostic tests, and therefore are given inappropriate medications, commence futile avoidance measures, or embark on potentially harmful elimination diets [3]. Skin or blood testing to determine the involvement of slgE in the aetiology of the signs and symptoms can provide objective information that is essential prior to treating and managing these patients.

Considering the difficulties in distinguishing between allergic symptoms and those symptoms which are non-allergic in origin, any practising physician within primary care is faced with several important questions:
1. To what extent does allergy contribute to the presence of symptoms (e.g. wheeze, rhinitis, eczema)?

2. Does allergy contribute to the severity of symptoms?

3. Will the symptoms persist or resolve?

Family history of allergic disease may help in this decision-making process. A history of other allergic diseases within an individual may also contribute. For example, young children with a diagnosis of

\section{Box 1}

(a) Atopy is the propensity to produce specific lgE (slgE) antibodies upon caposure to allergens in the envifon $e_{n}$, with subsecienc cillic a' symptoms.

(i) A cilleryic reaction is an immunologicallydetermined clirical reaction to an identitied suustance or allergen. IgEmediated allergy means that the immunological mechanism is related to slgE.

(c) Sensitisation means that slgE antibodies are formed upon allergen exposure, and can be determined in the blood or in the skin.

(d) Total and specific IgE: total lgE means the total amount of the immunoglobulin IgE present in blood, irrespective of what these IgE molecules may bind to; slgE means specific IgE antibodies binding to particular and identifiable allergens.

(e) Sensitivity and specificity: sensitivity is defined as the ability of a test to identify patients with the disease, that is the proportion of correctly identified test-positive patients in relation to the total number of truly allergic patients; specificity defines the ability of the test to identify only truly allergic patients and exclude those with no allergy, that is the proportion of negative patients in relation to the total number of patients without allergy. 
atopic dermatitis have been shown to have a $40 \%$ probability that they will develop asthma later in life [4]. In this context, questions to ask the patient would include:

1. Have you had hay fever?; have you been tested for allergy before and were the tests positive?; and has a doctor already diagnosed you with rhinitis or asthma?

2. Does anybody in your family suffer from asthma, hay fever or eczema?

3. Are symptoms worse during any particular time of the year?; and have you had symptoms during the last 12 months?

4. Are your problems associated with any particular organ?

5. Do your symptoms get worse when in contact with dust and during cleaning the house?; or when you're in contact with cats, dogs, pollens, or mouldy environments?

6. Do other substances like tobacco smoke, or odours from flowers and perfume, increase your problems?

In addition, more recent publications suggest that information regarding obesity, physical inactivity and time spent indoors may add to the precision of the diagnosis $[5,6]$.

\section{IgE antibody-rnediatec'allergiccliseäse}

IgE antibody-mediated allergic diseases may progress along a certain pathway; in the young child this is sometimes called the "Allergic March", with eczema and gastrointestinal symptoms followed by respiratory problems. The corresponding formation of slgE to exposed allergens in the susceptible individual frequently starts with foods (e.g. hen's egg, cow's milk, peanut, soy and wheat), followed by the inhalant allergens like dust mite, cat dander, and later pollen [7-9]. Pollen-driven allergy in the respiratory tract (but also to some structurally related foods) may further complicate the picture $[10,11]$. In contrast, some individuals will outgrow their allergy and become tolerant and stop getting symptoms to the allergen upon exposure [12]. Nevertheless, in each individual case presenting with symptoms resembling allergy, the presence or absence of allergy can be difficult to determine without an IgE antibody test [1]. However, it is essential to emphasise that the test results need to be interpreted in the context of the clinical presentation [3,13-15].

\section{Allergy in relation to exposure to allergens}

Allergy development and formation of slgE is a cumulative process and should not be regarded as an "all or nothing" phenomenon. Thus, all individuals (even if they are sensitized) have a certain level of tolerance to exposure to an offending substance, but when exposure is increased symptoms may become evident.

When evaluating individual allergen exposure, it is also important to understand that several allergens may have components of homologous structures, and consequently the personal allergen load may be higher than that which appears immediately obvious. For example, birch alder and hazel contain similar structures, as do different grasses. There is also a link between insects and shell fish, and between latex, and banana, kiwi and avocado. Furthermore, pollen allergy may manifest as a clinical reaction to certain vegetables due to chemical or immunological similarity in the structure of some of the components and molecules of the food compared with ine pollen (in the great majority of cares his simitar ty is related by taxonoiny, althougl cross reactivity may occur ats (betrween species which are not closely related) $[10,11]$. In 's' in cos, the different allergens can work i (i) concert, causing the allergy and producing functionally higher slgE levels. There is a link between allergy to birch, alder, and hazel pollen, as compared with allergy to hazelnut, apple, pear, stone fruits, tomato and almond. Similarly, mugwort crossreacts with celery, carrot and certain spices, whereas grass pollen is associated with reactions to tomato and peas, including peanut and wheat, and also melon, watermelon and orange and ragweed with melon and banana. Furthermore, in cases where substantial cross reactivity may be present there is good information available to which the reader is referred $[10,11]$, but a referral to an allergist may be warranted.

One clinical example easily recognized is the relation between pollen allergy and reactions to certain foods manifesting as the so-called Oral Allergy Syndrome (OAS). In OAS, the symptoms occur preferentially in the mouth and pharynx with clinical signs of oral itching, lip swelling, labial angio-oedema and glottal oedema frequently seen to fruits, vegetables and spices. These reactions may particularly manifest themselves during the pollen seasons of birch, grasses and weeds, when patients are exposed to increased amounts of allergen. 
Being exposed to an allergen at different doses may be illustrative. It has been shown that children with dust mite allergy who have symptoms at sea level where dust mite exposure is high may clinically improve by moving to higher altitudes where dust mites are not as prominent [16]. Thus, when it is practical, avoiding allergen exposure for sensitive individuals is a useful tool in the management of the patient.

In contrast, there has been recent debate as to whether very high exposure to an allergen can actually decrease reactivity and permit development of tolerance in the patient and induce protection of the allergic subject $[17,18]$. In fact, heavy exposure to allergen, particularly from pets (such as cats and dogs), may preferentially drive other immune responses rather than slgE formation, and therefore result in less allergy $[18,19]$.

\section{Allergic sensitization and IgE antibodies in clinical context}

The level of total IgE depends on genetic control of IgE production as well as on the synthesis of specific IgE antibodies (slgE) particularly after a parasite infestation. Total IgE levels have some - albeit weak - relation to the severity oi atopy in atopic dermatitis; they aloo es $\mathrm{E}$ 10 some extent associated wito tion severity of allergy in asthmo rif in ts. however, normal total IgE value: vary with age and seles:fiar, of the reference population. There is a considerable overlap between non-atopic and atopic patients, and also between the different allergic diseases, thus making the interpretation of the total IgE levels in an individual patient uncertain. Further, they do not give much information regarding the presence and progress of allergic disease. In contrast, slgE are specifically produced following exposure of a susceptible individual to an allergen. Generally they reflect the specific allergy and clinical reactivity in a patient. The slgE are present in blood and bind to allergy cells and can thereby cause a clinical reaction after subsequent allergen exposure. Thus, slgE can be regarded as a risk factor for clinical allergy in the respiratory tract, skin, and gastrointestinal tract, upon exposure to the allergen.

In patients with rhinitis, asthma and/or atopic dermatitis, and stinging insect anaphylaxis, case history and physical examination - although contributing to the diagnosis - are on their own not adequate to diagnose the presence and extent of allergy. Such cases require a firm confirmation of the presence of a slgE-mediated aetiology $[20,21]$.
In fact, studies have shown that when clinicians use only the history and physical examination, the accuracy of their diagnoses rarely exceeds $50 \%$ [23].

The addition of slgE antibody tests improves the accuracy of diagnosis, and allergy testing should be considered as an essential adjunct to the clinical history and physical examination, as is the case in other disease areas like hypercholesterolemia and diabetes, where all information is evaluated together which then guides therapeutic decisionmaking. In allergy, clinicians frequently follow a 'trial and error' process, by progressing directly from patients presenting signs and symptoms to pharmacotherapy. Empirical management may result in inadequately controlled symptoms and repeat office visits, as well as unnecessary referrals. However, in some patients with an intermediate or high probability of allergy, as assessed by history and physical examination, the IgE antibody results may prove negative. In such cases, extensive analysis of the case history and empirical drug therapy is warranted with further testing reserved for those who do not respond adequately [22].

\section{lgE - a quantitative approach}

Recent studies stigsest that for the individual potient, eren higher precision can be achieved by Utilizing a quantitative approach to the IgE antibody results rather than a dichotomous "yes/no", approach. This was first demonstrated in food allergy by Sampson and his colleagues [24,25]. A higher IgE antibody value implies that the subject will react with symptoms upon exposure and the patient can be diagnosed without further measures. In contrast, a lower, albeit still "positive", value is not really predictive, whether or not the subject will exhibit a clinical reaction upon exposure. This means that referral to an allergist for a challenge procedure may be considered. A still lower value implies rather low probability that the subject will react upon exposure, and the food should not be considered as a likely problem for symptoms (see Figure 1). However, in cases with a very convincing history despite a low slgE value, further investigation is necessary. One difficulty with different food allergens is that they vary in their potency and show different values for when a clinical reaction is likely to occur when the individual is exposed (Figure 1). Thus, it is necessary for the physician to get a feeling for the probability curve related to the allergy in question. A further complication is that the threshold values also differ by age [26-29]. Thus, patients with 

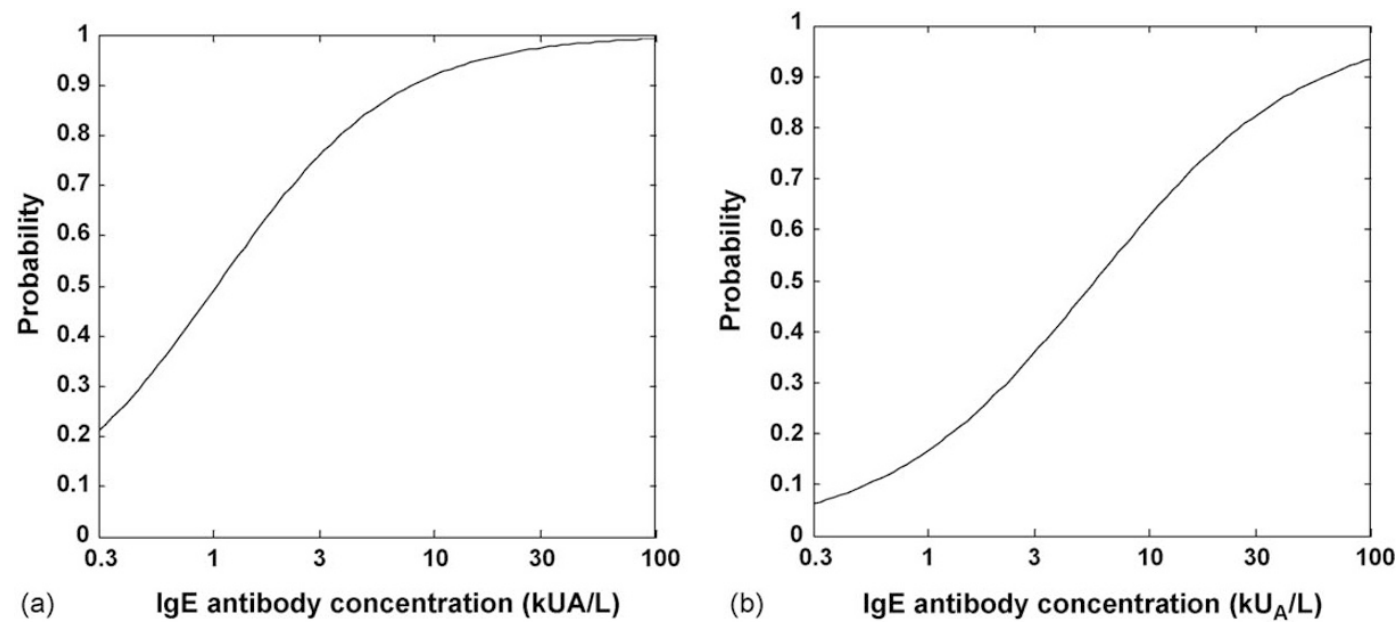

Figure 1 Probability of reacting clinically after eating (a) hen's egg and (b) cow's milk at a given slgE level. Data extracted from $[24,25]$. From a practical point of view levels indicative of a very high likelihood of clinical reactivity may be identified as a navigation tool for when the allergen should be absolutely avoided, when the patient needs a referral to a specialist for diagnosis by challenge, and when the allergen is not very likely to be the allergy culprit.

food allergy generally may be better managed by considering referral to an allergist.

For inhalant allergy, implementing slgE testing in the primary care setting may increase the accuracy of the diagnosis and the management of the patient to a considerable extent. In partic utiar, a number of uncertain and equivecal cases can be given a firm diagnosis [30 if]. Furthermore, quantitativg ignalit loc dy patterns similar to those described for food allergy hive teen, evealed for a variety of allergens, providing information on the contribution and extent to which allergy contributes to the expression of symptoms $[19,23,32]$. It is well known that most allergic individuals showing symptoms have slgE to several allergens and rarely only to one. Reports from a prospective birth cohort study demonstrate that a single positive slgE test was seldom associated with clinical allergic disease in the context of exposure of the patients to allergen. In contrast, four or more positive slgE tests out of a total of 14 common allergens, or a sum of slgE above $34 \mathrm{KU}_{\mathrm{A}} / \mathrm{L}$ to the same allergens, had a $75 \%$ likelihood of identifying those with allergic disease [33]. In practical terms, this implies that to obtain an adequate diagnosis in primary care, allergy tests should be performed to the most common allergens evident in the patient's environment, and that quantitative information should be gained and summated (see below and also Figure 3).

In a recent study from our group we reported that not only was being sensitised a risk factor for hospital admission of adults with asthma, but that the risk increased with increasing specific lgE levels [34]. Further analysis of our data on school children showed that the sum of mite, cat and dog specific IgE was associated with on increased risk of hospital admission with an as thma exacerbation, such that is silin of $10 \mathrm{~b} \mathrm{U}_{\mathrm{A}} / \mathrm{L}$ slgE increased the risk atnos 2.5 times and a sum of $30 \mathrm{KU}_{\mathrm{A}} / \mathrm{L}$ increased the risk thre efold. This corresponds to a $30-40 \%$ probahility or being admitted to hospital due to an astnma exacerbation (Figure 2, solid line) [35].

Since asthma exacerbations are well known to follow virus infections, much focus has been placed on the role of virus infections and the susceptibility of asthmatic subjects to such infections. Recent information points to extensive synergistic effects of virus infections and allergic inflammation both in children and adults [34-36]. Elevated slgE levels and exposure to allergens appear to interact with virus infections by strongly increasing the probability of hospitalization amongst childhood asthmatics over and above that of the slgE alone. In these cases therefore, a sum of $3 \mathrm{KU}_{\mathrm{A}} / \mathrm{L}$ of slgE may correspond to a $60 \%$ probability, and a sum of $30 \mathrm{KU}_{\mathrm{A}} / \mathrm{L}$ of more than $80 \%$ probability, of hospital admission (Figure 2, dotted line) [35]. Therefore, during periods of virus infections, the patient's allergic status should be especially well managed and evaluated, and measures undertaken both to decrease risk of allergen exposure in relation to the slgE levels and possibly to include prescription of increased doses of inhaled corticosteroid.

Recent information demonstrates that using the sum of slgE antibody levels against the most common inhalant allergens provides the answer to the question as to whether allergy contributes 


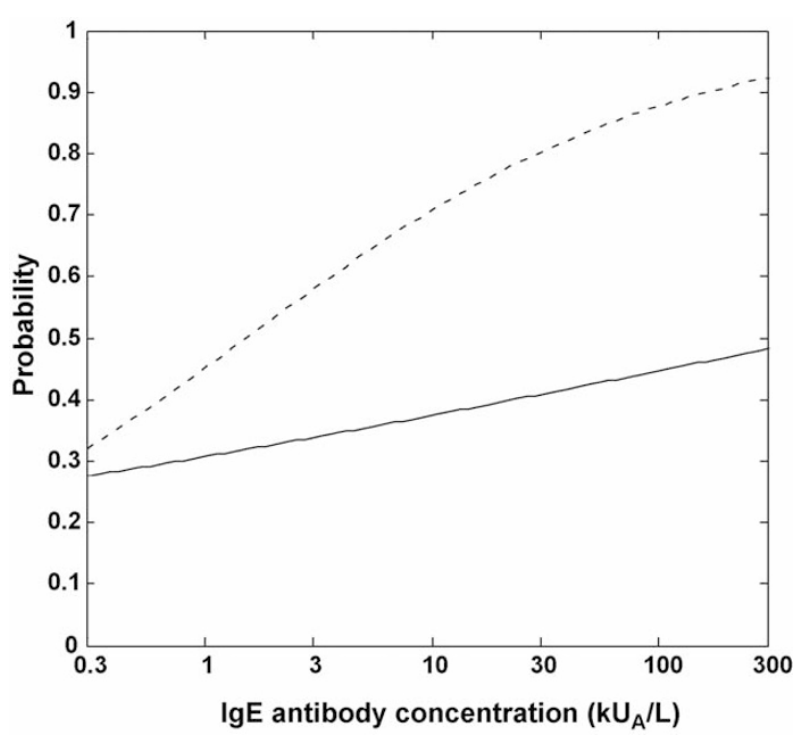

Figure 2 Probability of asthma patients needing emergency care. The solid line is the probability in relation to the sum of slgE to cat, mite and dog with no virus infection. The dotted line is the relation to slgE level and virus infection. Data recalculated from [35]. From a practical point of view this information indicates the need to understand allergen sensitization and allergen exposure in order to prescribe avoidance measures and increased pharmacotherapy and management during a virus infection season.

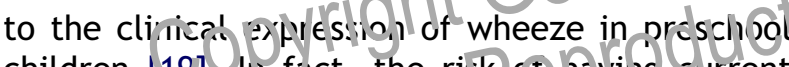
children [10]. In fact, the riv (it) ia ving eurrent or persistent wheeze and imparred lung function in children increases with increasing levels of slgE antibodies, whereas total IgE does not provide such information. As an example, this corresponds to a three-fold increase in the risk of symptomatic wheeze at $10 \mathrm{KU}_{\mathrm{A}} / \mathrm{L}$ slgE to the sum of cat, dog and mite, compared to those without such slgE, and a four-fold increase at $30 \mathrm{KU}_{\mathrm{A}} / \mathrm{L}$ (cf Figure 3). Evaluation of the size of the Skin Prick Test wheal may also give similar information provided that the testing and interpretation is carefully standardized. This has to be done in each clinic and may be difficult in clinical routine practice. In practical terms this means that patients with allergic asthma should be investigated by their slgE levels, and subsequent measures should be employed to optimize pharmacotherapy by giving inhaled corticosteroids as well as antihistamines in combination with attempts to decrease the allergen exposure. Consequently a low or negative slgE level indicates the need for an alternative "non-allergy" treatment.

Many people want to know whether their disease will persist or resolve. Data has shown that the persistence of wheeze at age 5 years can be predicted using slgE antibody levels at age 3 years (more than a two-fold increase in risk per log- unit increase of slgE antibodies) [19]. In practical terms, this means that in a young child with wheeze there are several options available for improving the accuracy of the diagnosis - the clinical history with information on family history, family smoking habits and other environmental exposures, all adding a certain level of risk of the diagnosis in conjunction with the level of slgE. For example, $10 \mathrm{KU}_{\mathrm{A}} / \mathrm{L}$ of slgE in the presence of a positive family history will give a probability of current wheeze of about $90 \%$, corresponding to a 30 -fold risk. The same $10 \mathrm{KU}_{\mathrm{A}} / \mathrm{L}$ of slgE, even with a negative family history, will give a probability of current wheeze of $65 \%$, corresponding to an eight-fold increased risk (c.f. Figure 3 ). In this context, slgE to food must not be neglected even if the symptom is asthma $[37,38]$. Such information, together with the patient's case history, should allow the physician to reveal the likelihood of allergy and exposure to a specific

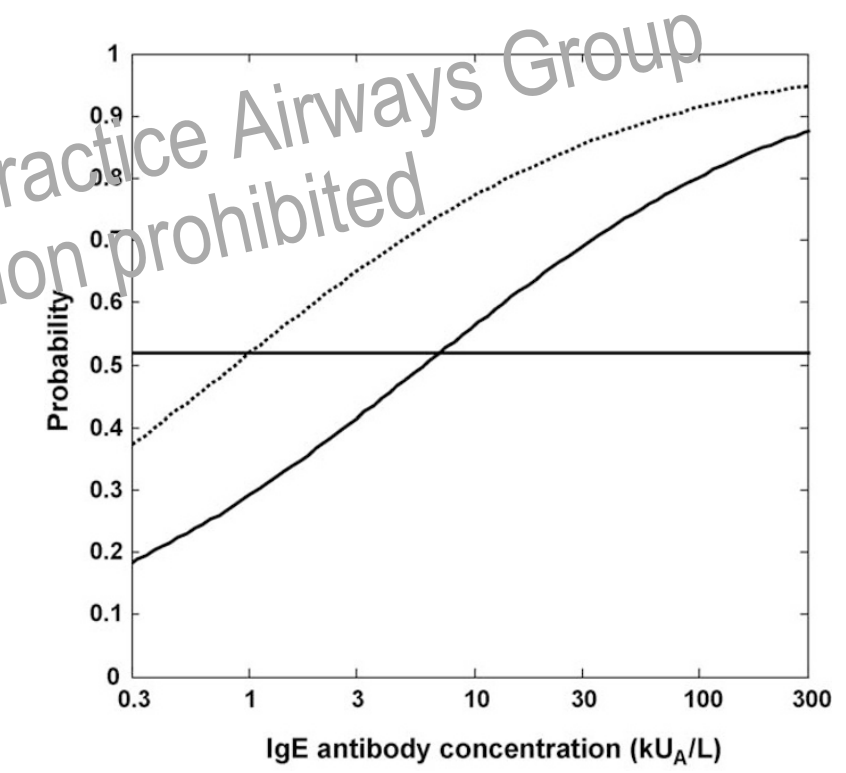

Figure 3 Probability of suffering from current wheeze in relation to the sum of slgE level to cat, mite and dog. The bold horizontal line indicates the probability after obtaining a good case history alone. The solid curved line indicates the probability given by the levels of slgE alone. The dotted line indicates the probability given by using the two sets of information in combination (data recalculated from [19]). From a practical point of view, this information will give a higher precision of diagnosis as to whether the wheeze is of an allergic or non-allergic nature with or without the use of a good case history. Note that a low slgE level decreases the probability despite a positive case history, whereas a high slgE level strongly indicates that the wheeze is of an allergic nature. 
allergen as being the driver of symptoms and disease, and allow the physician to adopt the appropriate therapy accordingly. For instance, it is not likely that a patient with very low slgE level to relevant allergens would benefit from inhalant antihistamine and corticosteroids.

In situations when the allergy is expected to disappear or is already fading away, slgE antibody determinations may also be particularly useful. Declining levels of slgE antibodies can be taken as a marker of decreased allergen exposure, or an emerging allergen tolerance. This has been well documented in children with food allergy, where a high $\left(>30 \mathrm{KU}_{\mathrm{A}} / \mathrm{L}\right.$ ), slowly decreasing (over more than 12 months) slgE antibody value indicates that tolerance may not be evolving, whereas a moderate to low value (approx $10 \mathrm{KU}_{\mathrm{A}} / \mathrm{L}$ ), decreasing by more than $75 \%$ in 12 months, is highly predictive of evolving tolerance [39]. In contrast, total IgE does not give such information.

\section{Performance of allergy tests}

The diagnostic performance of an allergy test - i.e. its ability to detect an allergic aetiology - is usually expressed as its clinical sensitivity and specificity using an arbitrary cut-off value as compared to a doctor's diagnesis. Soor seilsitivity and specificity result: for ty lancibody tests compared bo't W/thloctor's diagnosis and with skin prick tescing have beer edcumented for a variety of allergens using different methodologies [40]. Data for the ImmunoCAP system includes clinical documentation in thousands of patients in more than 3000 peer-reviewed publications; values above $90 \%$ sensitivity, specificity, and positive predictive value have been demonstrated for this system [41]. Other systems have been less extensively evaluated, and frequently show less accurate results although they usually compare themselves with ImmunoCAP [43-45].

To use quantitative IgE information in a clinical setting, a system for highly precise, reproducible, and accurate determination of the IgE antibody levels is essential [42]. It has to be emphasized that this approach is not generally applicable to all IgE antibody determination systems available. It has only been documented with one testing modality (ImmunoCAP) after very careful standardization [42], and therefore data from that system has been used for developing quantitative probability models attributing the risk of clinical disease; the models are only reliable for this system, and cannot be generalised, since the IgE results obtained using other test systems may differ significantly
$[43,44]$. Therefore, prescribing clinicians and testing laboratories need to be aware of possible discrepancies in the results from different systems [45]. Similar results may be obtained using extremely carefully performed skin prick tests with high quality extracts and precise assessment of the wheal size. However, this is unlikely to be applicable to a general clinical setting, where skin tests are performed on a routine basis by different operators using a range of different allergen extracts $[19,46]$.

\section{Conclusions}

About $50 \%$ of patients with symptoms from the respiratory tract and the skin may have an underlying allergy with specific triggers. In primary care there is the concept that confirmation of allergy with slgE is only necessary in situations where there is diagnostic uncertainty or in situations where avoidance of the offending allergen is possible and effective. There is a vast literature to show that specific diagnosis of allergic disease is complex and goe; bey ond taking a good case history and prysical examination. Allergy te-ing wifis a good system reveals the aillergic condition of the patient and identifies the allergy tripges that provide good information abouf theldisease, its progression, and how to manage it. Determination of slgE antibodies can give information on the aetiology of the disease, whereas little such information can be gained from measuring total IgE levels. Determination of the presence of slgE and sensitization in a patient can be obtained by using different test modalities. For the information needed about the allergy status of a patient and the importance of different allergy triggers, tests for slgE to the most prominent individual allergens in the patient's environment may be used utilizing a blood test or Skin Prick Test. A highly reproducible blood test system has advantages regarding standardization of allergens and interpretation of the results between users and over time. This information has previously been used as a dichotomous "all or nothing" phenomenon. However, utilizing slgE antibody results as a continuous variable provides additional information to the clinician beyond just the positive and negative results. We have demonstrated a model by which one can determine: the likelihood of the presence of allergy as a contributor to the symptoms and disease; an allergen or allergens as the dominant triggers of the symptoms; and the probability of the disease resulting in hospital admission. 


\section{Conflict of interest}

Staffan Ahlstedt is employed by Phadia $A B$ and is associated with the Karolinska Institute.

Clare Murray has no conflict of interest.

\section{References}

[1] Kurukulaaratchy RJ, Matthews S, Arshad SH. Defining childhood atopic phenotypes to investigate the association of atopic sensitization with allergic disease. Allergy 2005;60(10):1280-6.

[2] Agency for Health Care Research and Quality (AHRQ). Evidence Report/Technology Assessment No 54. Management of allergic and Nonallergic Rhinitis. 2004 [cited Accessed Feb. 6 2004]; Available from: http:/ /www. ahrq.gov/.

[3] Platts-Mills TAE. et al. Improving the diagnosis and care of allergic diseases, submitted.

[4] Kulig $M$, et al. Long-lasting sensitization to food during the first two years precedes allergic airway disease. The MAS Study Group, Germany. Pediatr Allergy Immunol 1998;9(2):61-7.

[5] Platts-Mills TA, et al. Is the hygiene hypothesis still a viable explanation for the increased prevalence of asthma? Allergy 2005;60(Suppl 79):25-31.

[6] Luder E, et al. Body mass index and the risk of asthma in adults. Respir Med 2004;98(1):29-37.

[7] Sasai K, et al. Early detection of specific IgE antibody against house dust mite in children at risk of allergic disease. J Pediatr 1996;128(6):834-40.

[8] Sigurs N, et al. Appearance of atopic disease in relation \pm 0 serum IgE antibodies in children followed up from in $^{2}$ : iv $_{1}$ 4 to 15 years. J Allergy Clin Immu 10 15?;: (i) 75-63.

[9] Wahn U, Bergmann RI- V Cikel $h$ Farly life markers of atopy a ic asth na. (Clin Ex, Aliergy 1998;28(Sunp.) ) 2 ) discussion 3? - $t$.

[10] Jenkins JA, et al. Structura veldtedness of plant food allergens with specific reference to cross-reactive allergens: an in silico analysis. J Allergy Clin Immunol 2005;115(1):163-70.

[11] Weber RW. Patterns of pollen cross-allergenicity. J Allergy Clin Immunol 2003;112(2):229-39, quiz 240.

[12] Sicherer SH, Teuber S. Current approach to the diagnosis and management of adverse reactions to foods. J Allergy Clin Immunol 2004;114(5):1146-50.

[13] Host A, et al. Allergy testing in children: why, who, when and how? Allergy 2003;58(7):559-69.

[14] Eigenmann PA. Breaking frontiers for better early allergy diagnosis. Allergy 2004;59(9):895-6.

[15] Eigenmann PA. Diagnosis of allergy syndromes: do symptoms always mean allergy? Allergy 2005;60(Suppl 79):6-9.

[16] Boner AL, et al. Influence of allergen avoidance at high altitude on serum markers of eosinophil activation in children with allergic asthma. Clin Exp Allergy 1993;23(12):1021-6.

[17] Hesselmar B, et al. Does early exposure to cat or dog protect against later allergy development? Clin Exp Allergy 1999;29(5):611-7.

[18] Platts-Mills T, et al. Sensitisation, asthma, and a modified Th2 response in children exposed to cat allergen: a population-based cross-sectional study. Lancet 2001;357(9258):752-6.

[19] Simpson A, et al. IgE antibody quantification and the probability of wheeze in preschool children. J Allergy Clin Immunol 2005;116(4):744-9.
[20] Wood RA. The diagnosis of allergy: why is it so difficult? Ann Allergy Asthma Immunol 2003;91(1):1-2.

[21] Biermann CW, Pearlmann DSE. Allergy, Asthma and Immunology from Infancy to Childhood. 3rd ed. Phila: WB Saunders Co.; 1995. p. 403.

[22] Gendo K, Larson EB. Evidence-based diagnostic strategies for evaluating suspected allergic rhinitis. Ann Intern Med 2004;140(4):278-89.

[23] Williams PB, et al. Are our impressions of allergy test performances correct? Ann Allergy Asthma Immunol 2003;91(1):26-33.

[24] Sampson HA, Ho DG. Relationship between food-specific IgE concentrations and the risk of positive food challenges in children and adolescents. J Allergy Clin Immunol 1997;100(4):444-51.

[25] Sampson HA. Utility of food-specific IgE concentrations in predicting symptomatic food allergy. J Allergy Clin Immunol 2001;107(5):891-6.

[26] Boyano Martinez $T$, et al. Validity of specific IgE antibodies in children with egg allergy. Clin Exp Allergy 2001;31(9):1464-9.

[27] Garcia-Ara C, et al. Specific IgE levels in the diagnosis of immediate hypersensitivity to cows' milk protein in the infant. J Allergy Clin Immunol 2001;107(1): 185-90.

[28] Roberts G, Lack G. Diagnosing peanut allergy with skin prick and specific IgE testing. J Allergy Clin Immunol 2005;115(6):1291-6.

[29] Eigenmann PA. Are specific immunoglobulin E titres reliable for prediction of food alie'gy: Cl $n$. Ixp Allergy 2005;35(3):247-9.

[30] Crobach $N$ J $\in t$ al The alagnosis of allergic rhinitis: $\mathrm{Cn} v \mathrm{~N}$ combine the medical history with the results of radioallergosprbeint kes cs and skin prick tests. Scand J Prim Health Cire 1998; $6(1,1): 30-6$.

[3] ['U ¿n Tauleria E, Vignati G, Guedan MJ, Peterson CJ. The utility of specific immunoglobulin $E$ measurments in primary care. Allergy 2004;78:35-41.

[32] Soderstrom L, et al. A further evaluation of the clinical use of specific IgE antibody testing in allergic diseases. Allergy 2003;58(9):921-8.

[33] Wickman $M$, et al. Quantitative analysis of IgE antibodies to food and inhalant allergens in 4-year-old children reflects their likelihood of allergic disease. Allergy 2005;60(5):650-7.

[34] Green RM, et al. Synergism between allergens and viruses and risk of hospital admission with asthma: case-control study. BMJ 2002;324(7340):763.

[35] Murray CS, et al. A study of modifiable risk factors for asthma exacerbations: virus infection and allergen exposure increase the risk of asthma hospitalization in children. Thorax 2005.

[36] Heymann PW, Platts-Mills TA, Johnston SL. Role of viral infections, atopy and antiviral immunity in the etiology of wheezing exacerbations among children and young adults. Pediatr Infect Dis J 2005;24(11 Suppl):S217-22, discussion S220-1.

[37] Roberts G, et al. Food allergy as a risk factor for lifethreatening asthma in childhood: a case-controlled study. J Allergy Clin Immunol 2003;112(1):168-74.

[38] Wang J, Visness CM, Sampson HA. Food allergen sensitization in inner-city children with asthma. J Allergy Clin Immunol 2005;115(5):1076-80.

[39] Shek LP, et al. Determination of food specific IgE levels over time can predict the development of tolerance in cow's milk and hen's egg allergy. J Allergy Clin Immunol 2004;114(2):387-91. 
[40] Yunginger JW, et al. Quantitative IgE antibody assays in allergic diseases. J Allergy Clin Immunol 2000;105(6 Pt 1):1077-84.

[41] Poon AW, Goodman CS, Rubin RJ. In vitro and skin testing for allergy: comparable clinical utility and costs. Am J Manag Care 1998;4(7):969-85.

[42] Ahlstedt S. Understanding the usefulness of specific IgE blood tests in allergy. Clin Exp Allergy 2002;32(1):11-6.

[43] Williams PB, et al. Analytic precision and accuracy of commercial immunoassays for specific IgE: establishing a standard. J Allergy Clin Immunol 2000;105(6 Pt 1):1221-30.
[44] Szeinbach SL, et al. Precision and accuracy of commercial laboratories' ability to classify positive and/or negative allergen-specific IgE results. Ann Allergy Asthma Immunol 2001;86(4):373-81.

[45] Hamilton RG. Responsibility for quality IgE antibody results rests ultimately with the referring physician. Ann Allergy Asthma Immunol 2001;86(4):353-4.

[46] Portnoy J. Diagnostic tests for allergies. Ann Allergy Asthma Immunol 2006;96:3-4.

Available online at www.sciencedirect.com

science@direct.

\section{Available online at http://www.thepcrj.com}

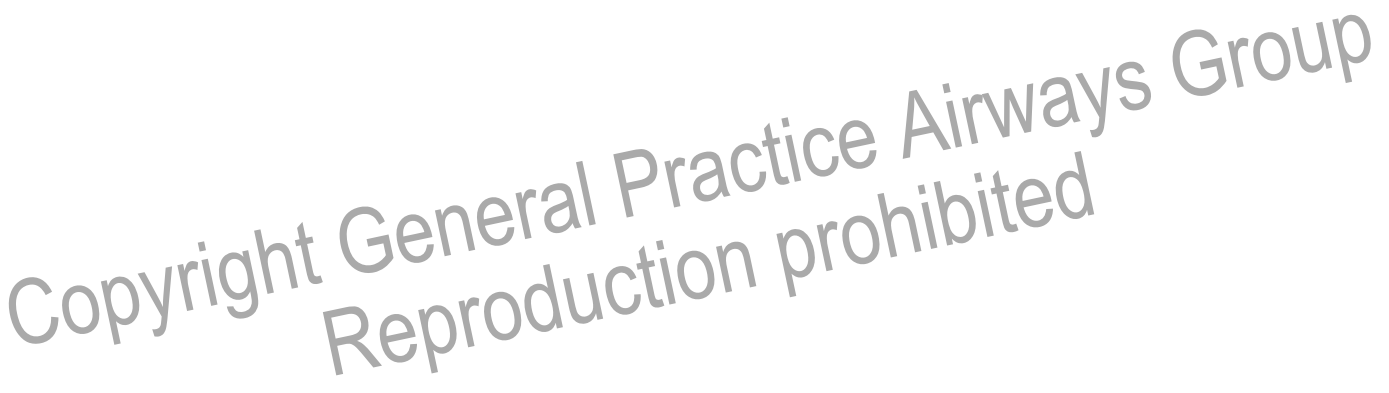

